\title{
INTOXICAÇÕES EXÓGENAS EM CLÍNICA MÉDICA
}

\author{
EXOGENOUS INTOXICATIONS IN CLINICAL MEDICINE
}

Renê Donizeti Ribeiro de Oliveira' \& João Batista de Menezes² $^{2}$

\begin{abstract}
${ }^{1}$ Residente em Imunologia. ${ }^{2}$ Médico Assistente de Toxicologia. Hospital das Clínicas da Faculdade de Medicina de Ribeirão Preto - USP. Correspondência: Prof. Dr. João Batista de Menezes. Unidade de Emergência do Hospital das Clínicas da Faculdade de Medicina de Ribeirão Preto/USP. Rua Bernardino de Campos, № 1000 - CEP: 14015-030 Ribeirão Preto-SP.
\end{abstract}

OLIVEIRA RDR \& MENEZES JB. Intoxicações exógenas em Clínica Médica. Medicina, Ribeirão Preto, 36: 472-479, abr./dez.2003.

RESUMO - Na primeira parte desta revisão, são discutidas as medidas gerais de tratamento das intoxicações exógenas (indução de vômitos, lavagem gástrica, administração de carvão ativado e de laxativos) e as medidas específicas de eliminação dos agentes tóxicos (diurese forçada e alcalinização da urina, hemodiálise e hemoperfusão e a utilização de antídotos e antagonistas). Na segunda parte, são discutidos os princípios do tratamento das mais freqüentes intoxicações exógenas em nosso meio (pesticidas agrícolas, depressores do sistema nervoso central e raticidas).

UNITERMOS - Envenenamento. Antídotos. Toxicologia.

\section{1 - INTRODUÇÃO}

O paciente intoxicado difere, em alguns aspectos, daqueles assistidos no cotidiano de um atendimento de emergência. As diferenças estão nos aspectos clínicos, patológicos e farmacológicos, e, também, no relacionamento médico-paciente.

Habitualmente, não se trata de pessoas doentes no sentido estrito da palavra. Na maioria dos casos, são pessoas saudáveis, que desenvolvem sintomas e sinais decorrentes do contato com substâncias externas e dos efeitos sistêmicos delas. As substâncias podem ser de uso industrial, doméstico, agrícola, automotivo, etc. Outras, são de uso humano, médico, na maioria, resultando em efeitos tóxicos pelo mau uso ou pelo abuso.

A tomada da história clínica, na intoxicação para tentativa de auto-extermínio, torna-se um desafio. Pouco se pode confiar nas informações acerca das substâncias utilizadas, das quantidades e do tempo de- corrido. O exame físico detalhado e repetido sistematicamente é o melhor método para o diagnóstico (muitas vezes, não o de certeza, mas o mais provável) e para a orientação do tratamento. Deve-se sempre confrontar a história obtida com os achados do exame físico. Se houver discordância, levar em consideração as informações do último.

A farmacologia da intoxicação é outro ponto peculiar. Não se podem aplicar os conceitos de farmacodinâmica ou farmacocinética ao paciente intoxicado. Um produto, em doses tóxicas, passa a ter efeitos outros que os habituais, em doses terapêuticas, pois passa a atuar em mecanismos moleculares diversos, muitos dos quais ainda desconhecidos. Conhecer o quadro clínico e o manejo das principais intoxicações é essencial àqueles que prestam assistência médica de emergência.

O laboratório é uma ferramenta de grande auxílio em toxicologia. Alguns compostos têm seus metabólitos identificados na urina. Outros, podem ser 
identificados no soro. A dosagem sérica da substância é um dado importante na classificação de gravidade da intoxicação por alguns compostos. Dosagens seriadas são importantes em intoxicações graves, sendo indicadores de resposta ao tratamento, bem como do momento em que o tratamento específico pode ser interrompido.

E, por fim, é no relacionamento médico-paciente que talvez se encontre a maior dificuldade no atendimento em toxicologia. São freqüentes os sentimentos de raiva e de desprezo, principalmente contra os que tentam auto-extermínio. Não nos cabe, neste espaço, discutir os motivos que levam nossos pacientes a buscar tal solução para seus problemas. Mas, muito menos, nos é permitido não lhes prestar a devida assistência médica e humanitária. Nem só de problemas físicos padecemos.

\section{2 - MEDIDAS GERAIS}

Avaliar os sinais vitais e mantê-los em parâmetros adequados é o manejo básico, que deve ser dispensado a todo paciente em um atendimento de emergência. Isso pode ser feito de modo a manter os mecanismos fisiológicos, ou auxiliando-os, como no caso da ventilação mecânica, e é valido para o paciente intoxicado. Devemos ainda, despender todo o esforço possível na tentativa de retirar do organismo a substância causadora da intoxicação. Para tal, dispomos de algumas medidas gerais, aplicáveis a quase todos os casos de intoxicação.

Êmese - O uso de xarope de ipeca ou de outro indutor de vômito não é medida terapêutica aceitável nos nossos dias, pelo potencial risco de dano aos tecidos, quando expostos à substância tóxica mais de uma vez, bem como pelo risco de aspiração, principalmente em casos de vias aéreas desprotegidas (coma ou convulsões).

Lavagem gástrica - Com poucas exceções, todos os pacientes intoxicados devem ser submetidos a sondagem nasogástrica e lavagem do conteúdo gástrico. O controle das convulsões e a proteção das vias aéreas, nos pacientes comatosos, são as precauções necessárias antes de se proceder à lavagem do conteúdo gástrico. As contra-indicações são os casos de ingestão de corrosivos (pela possibilidade de haver perfuração esofagogástrica) ou de compostos hidrocarbonetos (pela possibilidade de pneumonite, se hou- ver aspiração). Em adultos, a lavagem deve ser realizada com 150 a $200 \mathrm{ml}$ de água ou solução salina, aquecidos a $38, \mathrm{O}^{\circ} \mathrm{C}$. As lavagens devem ser repetidas até que se obtenha líquido claro.

Carvão ativado - Medida posterior à lavagem gástrica. Também deve ser realizada em todos os intoxicados, sendo as exceções as mesmas para a lavagem gástrica. Seu efeito é melhor dentro da primeira hora após a intoxicação. Dificilmente se consegue estabelecer a relação temporal entre a exposição e a chegada do paciente ao hospital, sendo assim, tal medida deve ser tomada em todos os casos de intoxicação. Seu mecanismo de ação consiste na absorção de compostos, não somente os presentes na luz intestinal, mas, também aqueles já absorvidos, como no caso de bases fracas ou no caso de substâncias com circulação enteroepática. As precauções para seu uso são as mesmas para a lavagem gástrica. A dose utilizada em nosso serviço é de $30 \mathrm{gr}$, em uma solução a $10 \%$ em salina, a cada 4 ou $6 \mathrm{~h}$. A primeira dose deve ser drenada do estômago após 30 min e as demais devem permanecer no trato gastrointestinal para eliminação com as fezes. O tempo de utilização depende da gravidade da intoxicação e da evolução do paciente, mas, habitualmente, não ultrapassa $72 \mathrm{~h}$. Devemos lembrar que o carvão ativado é inefetivo contra alguns compostos, entre eles: álcalis cáusticos, lítio, álcoois e sais de ferro. Tem pouca efetividade contra organoclorados e digoxina.

Laxativos - O principal utilizado é o manitol, em solução a 20\%. A dose utilizada é de 100 a $200 \mathrm{ml}$, até de $8 \mathrm{em} 8 \mathrm{~h}$, nas primeiras $24 \mathrm{~h}$. Sua utilização tem importância em associação ao carvão ativado, nos casos de compostos de elevada toxicidade, diminuindo a chance de absorção por reduzir o tempo de contato com o trato gastrointestinal

\section{3- MEDIDAS ESPECÍFICAS PARA ELIMINA- ÇÃO}

Diurese forçada e alcalinização da urina Compostos de eliminação renal podem ter sua depuração aumentada por simples hiper-hidratação e uso de diuréticos potentes. Uma condição especial de hiperhidratação é a realizada nas intoxicações graves por barbitúricos, quando também se provoca a alcalinização urinária, conhecida como esquema de Briggs:

- $500 \mathrm{ml}$ de solução de glicose a $5 \%+10 \mathrm{ml}$ de solu- 
ção de cloreto de potássio a 19,1 \%, via endovenosa, em duas horas, seguidos de

- $500 \mathrm{ml}$ de solução de glicose a $5 \%+10 \mathrm{ml}$ de solução de bicarbonato de sódio a $8,5 \%$, via endovenosa, em duas horas, seguidos de

- $500 \mathrm{ml}$ de solução fisiológica a $0,9 \%+10 \mathrm{ml}$ de solução de cloreto de potássio a $19,1 \%$, via endovenosa, em duas horas, seguidos de

- $500 \mathrm{ml}$ de solução de glicose a 5\% + 10 -ml de solução de bicarbonato de sódio a 8,5\%, via endovenosa, em duas horas, seguidos de

- $500 \mathrm{ml}$ de de solução de glicose a $5 \%+10 \mathrm{ml}$ solução de cloreto de potássio a 19,1\%, via endovenosa, em duas horas, seguidos de

- $500 \mathrm{ml}$ de solução fisiológica a $0,9 \%+10 \mathrm{ml}$ de bicarbonato de sódio a $8,5 \%$, via endovenosa, em duas horas. Repetir o esquema até quando for necessário.

Tal esquema deve ser evitado em pacientes com insuficiência renal, pelo grande aporte hídrico. Devemse obter dosagens de sódio, potássio e análise dos gases sangüíneos a cada $6 \mathrm{~h}$ durante a realização do esquema.

Hemodiálise e Hemoperfusão - Esses dois métodos não são indicados com frequiência, e, se mal indicados, podem causar maior morbidade ao invés de ajudar na recuperação do paciente. É essencial que se tenha o conhecimento, se o tóxico em questão é extraído por tais métodos. São indicados em casos graves, casos com dosagem sérica em níveis letais, deterioração progressiva do quadro clínico a despeito de terapia adequada e casos de compostos com toxicidade retardada. Podem ser utilizadas em intoxicações por álcoois, barbitúricos, salicilatos, lítio, arsenicais, paraquat, compostos de ferro, mercúrio e chumbo entre outros.

Antidotos e Antagonistas - Também têm indicações precisas. É importante que se conheçam as indicações e se pese a necessidade para cada caso. Além disso, devem ser conhecidas e respeitadas as doses para um composto, a fim de não se causar nova intoxicação ao paciente. Abaixo, listamos os principais compostos, com suas indicações.

\section{4 - INTOXICAÇÕES MAIS FREQÜENTES}

Seria impossível fazer uma abordagem adequada de todas as intoxicações em tão curto espaço. Sendo assim, priorizamos aquelas que são mais freqüentes em nosso meio.

\section{Intoxicações por pesticidas agrícolas}

\section{Inibidores da acetilcolinesterase}

Neste grupo, encontram-se os Organofosforados e os Carbamatos. São compostos inibidores da acetilcolinesterase, enzima responsável pela degradação da acetilcolina, presente nas fendas sinápticas do sistema nervoso autônomo, do sistema nervoso central e da junção neuromuscular. A acetilcolina exerce sua atividade através de dois tipos de receptores: nicotínicos, presentes na placa motora, no sistema nervoso central (medula) e nos neurônios pós-ganglionares do sistema nervoso autônomo; muscarínicos, presentes no sistema nervoso central (encéfalo) e nos neurônios pós-ganglionários do sistema nervoso autônomo.

Esses compostos se ligam com a acetilcolinesterase de forma muito mais estável que a acetilcolina. A ligação do carbamato se desfaz em minutos a horas, de forma que a enzima é regenerada em 24-48 h. A ligação do fosforado permanece por dias a semanas, tornando a enzima indefinidamente, se não for usado composto que desfaça a ligação.

Fosforados e carbamatos são absorvidos por qualquer via: oral, transdérmica, através de mucosas (gastrointestinal, genitourinária, conjuntiva) e parenteral. A via inalatória levaria a menor período de latência, enquanto a transdérmica, a de maior período. O quadro clínico se instala de minutos até $12 \mathrm{~h}$. Os sinais e sintomas são decorrentes dos diversos sítios onde ocorrerá excesso de acetilcolina. Os efeitos muscarínicos levam a salivação excessiva, lacrimejamento, liberação de esfíncter vesical, diarréia, vômitos, broncoconstrição e broncorréia e aumento do tônus vagal cardíaco (lentificação da condução nos nós SA e AV). Os efeitos nicotínicos incluem fasciculações, câimbras e fraqueza muscular (inclusive de musculatura respiratória), hipertensão, taquicardia, dilatação pupilar e palidez cutânea. Os efeitos no SNC incluem inquietação, labilidade emocional, cefaléia, tremores, sonolência, confusão, ataxia, psicose, convulsões e coma. As mortes decorrem, em sua maioria, de depressão respiratória associada à hipersecreção traqueobrônquica.

A dosagem da acetilcolinesterase sangüínea é de extrema valia nessas intoxicações, guardando relação com a atividade da enzima neural e muscular. Apesar disto, a recuperação dos níveis de colineste- 


\begin{tabular}{|c|c|c|}
\hline $\begin{array}{l}\text { Antilotos e } \\
\text { antagenistas }\end{array}$ & Dose-vin de utilixaçăo & Tipo de inbxiraçăo-indicaçŏes \\
\hline Atropina & $\begin{array}{l}\text { 0,01 0,05 mg/kg/dose (IM), (EV), (SC), repetidos conforme a necessidade. } \\
\text { Dose varível }\end{array}$ & $\begin{array}{l}\text { Irbeticidas Organofosforados e } \\
\text { Carb arretos }\end{array}$ \\
\hline Aall de Metieno & 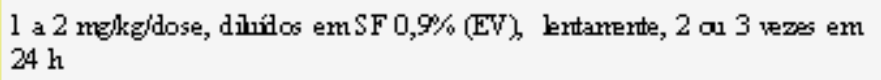 & Sulfonas, arnitien, ritratos e rüritos \\
\hline Aanl da Prissia & $\begin{array}{l}250 \mathrm{rg} / \mathrm{kg} / 24 \mathrm{~h} \text { por sonda duoderel em } 50 \mathrm{ml} \text { de maribl por dose, } 3 \\
\text { doses em } 24 \mathrm{~h}\end{array}$ & Tálo o \\
\hline Biperiden & 0,04 a 0,1 mg/kg/dose (IM) (EV), 4 vers ao dia & $\begin{array}{l}\text { Fenotizainas, Butioferonas, } \\
\text { Metocbpramida }\end{array}$ \\
\hline Calcio & Ghconato de cálcio a $10 \%$ (EV) várias vezes & Fhoretos, oxalatos \\
\hline Cloppromazina & 1 a 2 mg/kg/dia (IM), (EV), 3 on 4 veres ao dia & Arfetamires, LSD \\
\hline Dartwolene & $\begin{array}{l}1 \mathrm{rg} / \mathrm{kg} \text {, repetir at́ clegar a } 10 \mathrm{mg} / \mathrm{kg} \text { (EV), } 4 \text { a } 8 \mathrm{mg} / \mathrm{kg} \text { (WO), durante } 1 \\
\text { a } 3 \mathrm{dia} \text { em seguida ao uso ( } \mathrm{EV} \text { ), divididos em } 3 \text { doses }\end{array}$ & $\begin{array}{l}\text { Síndrome neuroḱptica mahigna, indurila } \\
\text { por anestésicos fenotiazícos }\end{array}$ \\
\hline Desferoxanire & $\begin{array}{l}20 \text { a } 60 \mathrm{rg} / \mathrm{kg} / \mathrm{dia}(\mathrm{EV}) \text { em S Fo,9\% ou (MM), dividilos em } 4 \text { a } 6 \text { doses de } \\
\text { á́ } 6 \mathrm{gem} 24 \mathrm{~h}\end{array}$ & Feno \\
\hline $\begin{array}{l}\text { Dimercaprol(BAL) } \\
\text { ou Demetal }\end{array}$ & $\begin{array}{l}3 \text { a } 5 \mathrm{rg} / \mathrm{kg} d \mathrm{~g}^{\circ} \text { e de } 4 / 4 \mathrm{~h}(\mathrm{MM}) \mathrm{nos} \mathrm{l}^{\circ} \text { e } 2^{\circ} \text { dias, de } 6 / 6 \mathrm{~h} 3^{\circ} \text { dia, de } 8 / 8 \mathrm{~h} \text {, } \\
4^{\circ} \text { dia, de } 12 / 12 \mathrm{~h} \text { do } 5^{\circ} \text { ao } 10^{\circ} \text { dia }\end{array}$ & $\begin{array}{l}\text { Asêrico, artinôrio, bis muto, auro, } \\
\text { mercúio, chumbo, rứulel }\end{array}$ \\
\hline EDTÁ cálcì & $\begin{array}{l}30 \text { a } 50 \mathrm{rg} / \mathrm{kg} \text { dia (EV) dinúdos em SFO, } 9 \% \text {, irfidilos eml a } 2 \mathrm{~h} \text { de } \\
\text { l2/12h por } 5 \text { dias }\end{array}$ & Clumbo, ưârì \\
\hline Etanol & $\begin{array}{l}6 \mathrm{a} 9 \mathrm{mlkg} \text { da sohŗão a } 10 \% \text { em S G5\% (EV) em rédia } 1,5 \mathrm{mllkg} / \mathrm{h} \text { ou } \\
0,5 \mathrm{mllkg} \text { de solyçăo a } 50 \% \text { (VO), de } 4,4 \text { hou de } 66 \mathrm{~h}\end{array}$ & Metanol, etilencelicol \\
\hline Frostignima & 0,03 a 0,08 mglog/dose (IM) repetir até atimgir $2 \mathrm{mg}$ em $24 \mathrm{~h}$ & $\begin{array}{l}\text { Articolinérjoos, antilepressinos tricíclicos, } \\
\text { fenotiazínicos, vegetais beladorados }\end{array}$ \\
\hline Fhmezeril & 0,5 a 2rg. Repetir se necessámo. Fazer pơ VIA (EV) & Berodiarepíricos \\
\hline Hidroxicobalanma & $\begin{array}{l}4 \mathrm{~g} \text {, dinúlos em SFO, } 9 \% \text { (EV), infusão de } 0,4 \mathrm{mg} / \mathrm{rm} \text {, repetir a dose, se } \\
\text { necessám. }\end{array}$ & Cianetos \\
\hline Kit para Cianetos & 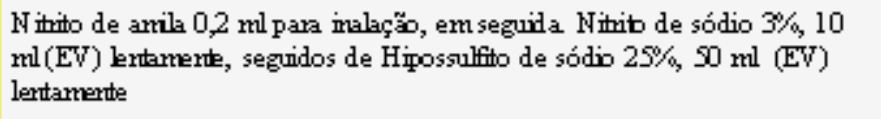 & Cianetos \\
\hline Nabuone & 0,01 rg/k (EV), (IM), (SC), repetir após algurs niutos, se necessám & O piáceos, ácilo valpróico, halotano \\
\hline Pericilanmina & $25 \mathrm{mg} / \mathrm{k} / \mathrm{dia}(\mathrm{VO})$ em 3 doses & Cobre, revoim, cromo, chunbo, zinco \\
\hline $\begin{array}{l}\text { Pemanganato de } \\
\text { Potássi }\end{array}$ & 1:10000 para lavagemgástrica & $\begin{array}{l}\text { Wrotina, fisostignire, alcalódes vários } \\
\text { anfetarinas, es tricrima, cianetos }\end{array}$ \\
\hline Pralidoxime & 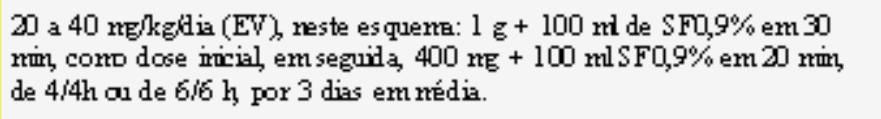 & Ire eticidas organofos forados \\
\hline Propanobl & $2-8 \mathrm{mg}$ (EV) dihúlos emSF0,9\% no réximo $1 \mathrm{mg} / \mathrm{nm}$ & Cocaina \\
\hline Terra de Füller & $\begin{array}{l}\text { Suspersão a } 30 \% \text {, } 500 \mathrm{~m} \text { (VO) como dose iricial em seguida } 200 \text { a } 300 \\
\text { ml(VO) de } 4 / 4 \mathrm{~h} \text { por } 2 \text { a } 3 \text { dis em rédia }\end{array}$ & Paraguat \\
\hline Vitanima $\mathrm{E}$ & 400 a $600 \mathrm{rg}$ (VO) dividilo em 3 doses & Paraquat \\
\hline Vitanima $\mathrm{k} 1$ & $\begin{array}{l}\operatorname{lng} / \mathrm{kg}(\mathrm{IM}) \text {, (EV), quando (EV) rão ultrapass ar l mginmin - Dose média } 40 \\
\text { mg/dia }\end{array}$ & Cumeríricos \\
\hline
\end{tabular}


rase a valores normais não deve ser o parâmetro para se interromper o tratamento, mas, sim, a ausência de manifestações clínicas de intoxicação, já que a recuperação desses níveis pode ocorrer em meses.

No tratamento, o uso de atropina é essencial, por ser um antagonista competitivo da acetilcolina, tanto no SNC quanto no SNA. A dose inicial para adultos é de 1 a $2 \mathrm{mg}$ para intoxicações por carbamatos e de 2 a $4 \mathrm{mg}$ para intoxicações por fosforados, por via endovenosa ou intramuscular. A dose a ser usada é empírica e será única para cada paciente. A dose inicial pode ser repetida em 5 a $10 \mathrm{~min}$, ou em infusão contínua, avaliando-se a necessidade de aumentá-la ou reduzí-la a cada administração, de acordo com o controle do quadro clínico, ou seja, o controle das secreções estimuladas pela atividade muscarínica. Uma vez ajustada a dose, esta deve ser mantida por, no mínimo, $24 \mathrm{~h}$. Os sinais de atropinização incluem midríase (o mais precoce), taquicardia e ruborização cutânea (este é útil no ajuste de dose). A retirada é lenta e gradual, por pelo menos $24 \mathrm{~h}$, devendo ser restituída, se reaparecerem os sintomas. Nos casos de intoxicação por fosforados, deve-se fazer uso de oximas. A Pralidoxima tem a capacidade de regenerar a colinesterase por ligar-se ao fosforado. A via de administração é endovenosa, devendo ser realizada em $30 \mathrm{~min}$, na dose de 20 a $40 \mathrm{mg} / \mathrm{kg}$, diluídos em solução salina a $0,9 \%$. A dose pode ser repetida em 1 a $2 \mathrm{~h}$, com dose máxima diária de $12 \mathrm{~g}$. Será suspensa somente quando forem abolidos todos os sintomas colinérgicos.

Organoclorados - São compostos estimulantes do SNC, com toxidade variável, quando ingeridos. Entre outros possíveis mecanismos de ação, atuam na membrana axonal, lentificando o fechamento dos cais de sódio voltagem-dependentes.

São absorvidos por via oral, transdérmica ou inalatória. Muitos são bastante lipossolúveis, levando a manifestações precoces do SNC. Esses compostos levam à diminuição do limiar convulsivo e ao estímulo do SNC. Sem dúvida, nas intoxicações por clorados, as convulsões são a principal ameaça, podendo abrir o quadro clínico. Podem ocorrer, também, náuseas e vômitos, hiperestesias e parestesias em face, língua e extremidades, cefaléia, tonturas, mioclonias, agitação e confusão. Febre de origem central não é infreqüente.

Não há exame laboratorial para identificação de organoclorados. Se o paciente apresentar rebaixamento importante do nível de consciência, proceder a intubação orotraqueal. Na presença de crises convul- sivas, pode-se utilizar Diazepam por via endovenosa. Pele e mucosas devem ser descontaminadas, com proteção da equipe que presta assistência. Hipertermia deve ser tratada com métodos físicos. Atentar para o desenvolvimento de necrose tubular aguda, por rabdomiólise decorrente das convulsões.

A Colestiramina, resina de troca iônica, não absorvível, deve ser utilizada ao invés de carvão ativado. A dose indicada é de $4 \mathrm{~g}$, por via oral, a cada $6 \mathrm{~h}$.

Piretróides - Análogos sintéticos das piretrinas são compostos de efeito rápido e letal para insetos, freqüentemente associados a Butóxido de piperonila. Como os clorados, prolongam o período de abertura dos canais de sódio voltagem-dependentes. São praticamente inabsorvidos pela pele. Suas manifestações principais decorrem da indução de manifestações alérgicas ao contato com a pele e mucosas. Quando ingeridos, podem levar a parestesias, náuseas e vômitos, tonturas e fasciculações. Lavagem gástrica e carvão ativado são suficientes.

Paraquat - Altamente solúvel em água, vendido em preparações de concentração em $20 \%$ em água. A inalação leva a nenhuma ou pequena intoxicação. A absorção a partir de pele ou mucosas intactas é mínima. Se ingerido, é rapidamente absorvido (30\% da dose) no intestino delgado. O pico plasmático ocorre de minutos a $2 \mathrm{~h}$ após a ingestão. Distribui-se para todos os tecidos do organismo, mas alcança maiores concentrações nos pulmões e nos rins, tecidos onde há transporte ativo da molécula. Aproximadamente 90\% do Paraquat absorvido é eliminado intacto por via renal, dentro de 12 a $24 \mathrm{~h}$.

A toxicidade do Paraquat encontra-se no efeito cíclico de redução de óxido, com produção de compostos tóxicos de oxigênio. Os radicais livres formados reagem com lipídeos da membrana celular, proteínas estruturais e enzimas, além da molécula DNA. O contato ocular pode levar a ceratite e conjuntivite. O contato repetido com a pele pode levar a dermatite e erosão unguenal. No trato gastrointestinal, podem ocorrer ulceração e corrosão orofaríngea, náusea e vômito, diarréia, hematêmese, disfagia, perfuração esofásiga, pancreatite e necrose hepática. Pode ocorrer necrose tubular aguda; no sistema respiratório, tosse, mediastinite, pneumotórax, hemoptise, hemorragia alveolar, edema e fibrose pulmonar; hipovolemia, choque e arritmias; convulsões, coma e edema cerebral. 
Os sintomas ocorrerão na dependência da dose ingerida. Se a ingestão foi de até $20 \mathrm{mg} / \mathrm{kg}$, as lesões são poucas e a recuperação ocorre. Se entre 20 e 40 $\mathrm{mg} / \mathrm{kg}$, há morte dentro de cinco dias, por acometimento gastrointestinal, renal e pulmonar. Se acima de $40 \mathrm{mg} / \mathrm{kg}$, há falência de múltiplos órgãos e morte em um a três dias.

O sucesso do tratamento depende do tempo de sua instituição. Consiste em lavar copiosamente pele e mucosa, se estas foram expostas. Para ingestão, pode ser empregado carvão ativado ou Terra de Füller em suspensão aquosa a $15 \%(15 \mathrm{~g} / 100 \mathrm{ml})$, na dose de 1 a $2 \mathrm{~g} / \mathrm{kg}$ de peso corporal. Manter hidratação e fluxo renal adequados. A suplementação de oxigênio pode potencializar os efeitos do Paraquat, só devendo ser realizada nos casos em que a hipoxemia é limitante para vida. Hemodiálise e hemoperfusão podem ser utilizadas para aumentar a eliminação. Nenhum, antídoto tem se mostrado efetivo. Outra tentativa é a utilização de compostos que previnam a formação de radicais livres, como vitamina $\mathrm{E} e$ vitamina $\mathrm{C}$. Tenta-se, também, reduzir a reação inflamatória, pulmonar, principalmente, com o uso de corticosteróides.

Paraquat pode ser dosado no sangue e na urina. O nível sérico tem estrita correlação com a morbidade. Muitos evoluem para o óbito.

\section{Medicamentos depressoras do SNC}

Benzodiazepínicos - Sedativos, hipnóticos e ansiolíticos, são medicações que atuam, aumentando a neutransmissão inibitória GABAérgica no SNC, através de aumento no número de canais abertos de cloreto.

São rapidamente absorvidos no intestino. O início da ação também é rápido. Medicações como o Midazolam e o Diazepam são intensamente lipofílicos, cruzando rapidamente a barreira hematoencefálica. Pelo mesmo motivo, redistribuem-se pelos tecidos adiposos, levando a meia-vida consideravelmente maior.

A ingestão aguda de altas doses de benzodiazepínicos pode levar a ataxia, fala empastada e sonolência. Raramente, doses elevadas podem levar a coma e depressão respiratória. Além do manejo básico, nos casos de depressão profunda do SNC ou de instabilidade hemodinâmica, pode-se fazer uso de Flumazenil. Este deve ser feito lentamente, a fim de não precipitar convulsões.
Barbitúricos - O principal uso deles, atualmente, é anticonvulsivante. Atuam, prolongando o período de abertura dos canais de cloreto, associados aos receptores GABA, Fenobarbital é o principal representante. Também apresentam elevada lipossolubilidade, com rápido início de ação e longa meia-vida. Metabolizado no fígado, é excretado, na sua maior parte, de forma inalterada, na urina.

O quadro clínico da intoxicação é um continuum, sendo a progressão limitada pela quantia ingerida. No início, há fala empastada, ataxia, cefaléia, nistagmo e confusão mental. Com a progressão, há os vários graus de coma, com perda total de reflexos. É comum haver hipotermia, depressão respiratória e da contratilidade miocárdica. A depressão miocárdica, associada a vasodilatação e depressão medular leva ao choque. $\mathrm{O}$ comprometimento cardiopulmonar é o principal responsável pelos óbitos na fase aguda. Tardiamente, os óbitos ocorrem por edema pulmonar, pneumonia e edema cerebral. A dose potencialmente fatal é de 6 a $10 \mathrm{~g}$.

Dispomos do método quantitativo para dosagem de barbitúricos. Além da utilidade evidente para o diagnóstico, a quantificação é importante para correlação com o quadro clínico e para monitoração do tratamento. Deve-se obter via aérea adequada, além de monitorização cardíaca adequada, com ECG seriados.

Fenobarbital é uma base fraca, sendo que, na presença de urina alcalina, haverá um maior transporte desse composto do plasma para os túbulos renais. O pH sangüíneo deve ficar em torno de 7,40-7,45, o que aumenta a excreção do Fenobarbital em cinco a dez vezes. Utilizar o esquema de Briggs. Hemodiálise é efetiva.

Antidepressivos tricíclicos - Os de primeira geração (Amitriptilina, Imipramina, Clomipramina e Nortriptilina) inibem a recaptação de noradrenalina e serotonina. Os de segunda geração (Fluoxetina, Paroxetina e Sertralina) são inibidores seletivos da recaptação de serotonina.

São rapidamente absorvidos a partir do trato gastrointestinal. Apresentam grande metabolismo de primeira passagem hepática, somente $10 \%$ é excretado de forma inalterada na urina. As intoxicações graves decorrem do abuso dos tricíclicos de primeira geração. Exercem efeito depressor na fibra miocárdica e levam a diminuição na resistência vascular periférica, resultando em hipotensão. Por efeito anticolinérgico, aumentam a frequiência cardíaca e predispõem a ta- 
quiarritmias. As convulsões ocorrem precocemente e têm curto período de duração, mas são potencialmente letais. Além dessas manifestações, podemos ter outras, como letargia, agitação, ataxia, movimentos coreoatetóicos, pele seca, constipação intestinal e retenção urinária.

Os tricíclicos de segunda geração são desprovidos dos efeitos anticolinérgicos, e não causam arritmias, hipotensão ou convulsões. Causam apenas sedação.

As intervenções iniciais incluem obtenção de via aérea segura, monitorização cardíaca contínua e estabilização dos sinais vitais. Os eventos letais ocorrem dentro das seis primeiras horas, em especial nas duas primeiras, com arritmias cardíacas, alterações de condução, convulsões, depressão respiratória ou hipotensão.

Manter o pH sanguiíneo entre 7,40 e 7,45 é útil para prevenir, mesmo que em parte, o efeito cardiodepressor do tricíclico. Para convulsões, utilizar benzodiazepínico ou barbitúrico; evitar Fenitoína, pela eficácia limitada e pelo efeito pró-arrítmico. A hipotensão deve ser tratada inicialmente com reposição volêmica de cristalóides. Caso refratária, pode-se usar Noradrenalina e Dobutamina. Evitar Dopamina, pelo efeito hipotensor.

Neurolépticos - Os de maior importância em toxicologia são os típicos, representados pelas fenotiazinas (Clorpromazina, Tioridazina) e pelas butirofenonas (Haloperidol). Têm efeito inibitório em receptores dopaminérgicos, colinérgicos, alfa 1 e alfa 2 adrenérgicos, histaminérgicos e serotinérgicos. São de uso oral exceto o Haloperidol, que também tem uso parenteral. Os efeitos da intoxicação por neurolépticos podem ser vistos em pessoas utilizando doses terapêuticas, não somente na overdose. Esses efeitos podem ser divididos em relacionados ou não ao SNC. Os não relacionados são: cardíacos (hipotensão, depressão miocárdica, prolongamento dos intervalos PR, QRS e QT, alterações inespecíficas de onda T e segmento ST, taquiarrítmias ventriculares e supraventriculares), gastrointestinais (boca seca, redução de motilidade e secreção, pseudo-obstrução, genitourinárias (retenção urinária, priapismo), midríase ou miose. Os relacionados ao SNC são: acatisia, distonia, confusão e alterações de memória, hipo ou hipertermia, parkinsonismo, diminuição do limiar convulsivo, sonolência e coma. Os efeitos no SNC são os mais comuns e mais importantes. Os níveis séricos não se correlacionam com a gravidade do quadro clínico. A
Cromatografia de Camada Delgada pode ser utilizada para análise qualitativa. Monitoração cardíaca e ECG seriado. Alcalinização da urina não é útil. Nos casos graves, pode-se utilizar Fisostigmina. Quando houver importantes sinais extrapiramidais, pode-se utilizar o Biperiden, um anticolinérgico, por via intramuscular, na dose de $0,08 \mathrm{mg} / \mathrm{kg}$ de $6 / 6$ horas.

A síndrome neuroléptica maligna é uma reação idiossincrásica, implicada aos neurolépticos típicos, caracterizada por hipertermia, rigidez muscular, acinesia, coreoatetose, flutuação do estado mental e alterações autonômicas (pressão arterial, frequiência cardíaca, padrão respiratório). Cursa com leucocitose, acidose metabólica, hipercalemia, elevação de enzimas hepáticas, creatinaquinase e creatinina. É diagnóstico de exclusão e exige ingestão recente das substâncias relacionadas. O tratamento consiste em abaixar a temperatura por métodos físicos. Dantrolene, para a hipertonia muscular e Bromocriptina, para acentuar e neurotransmissão dopaminérgica no SNC.

\section{Raticidas}

Cumarínicos - Com a proibição do uso de arsenicais como raticidas, os cumarínicos são os únicos raticidas atualmente comercializados. São inibidores competitivos da Vitamina $K$, interferindo na $\gamma$ carboxilação final dos fatores II, VII, IX e X, da qual ela é cofator. A intoxicação por tais compostos é assintomática por 12 a $24 \mathrm{~h}$, isto se deve ao tempo de vida-média do Fator VII. Somente após esse período, o paciente apresentará alterações da coagulação com sangramento em qualquer sítio, com as manifestações decorrentes de sua localização. Disso concluímos que não se deve esperar o paciente sangrar para iniciar o tratamento. Sempre que se tiver a suspeita de intoxicação por cumarínico e o paciente não apresentar sangramento, iniciar tratamento com Vitamina K 10mg, intramuscular, a cada 6 ou $8 \mathrm{~h}$. Após lavagem gástrica, utilizar Colestiramina, na dose de $4 \mathrm{~g}$ diluídos em $200 \mathrm{ml}$ de líquido, a cada $8 \mathrm{~h}$. O tempo de pró-trombina deve ser solicitado e repetido diariamente. Nos casos onde há sangramento identificado, pode-se recorrer ao uso de plasma fresco congelado, na dose habitual de até $20 \mathrm{ml} / \mathrm{kg}$ de peso corporal, a cada $6 \mathrm{~h}$. Esses compostos têm meia-vida longa ( $40 \mathrm{~h}$ para o Warfarin e 6 dias para a Femprocumona), por isso o tratamento com vitamina $\mathrm{K}$ deve permanecer por 24 a 48 h após a normalização do tempo de protrombina. 
OLIVEIRA RDR \& MENEZES JB. Exogenous intoxications in clinical medicine. Medicina, Ribeirão Preto, 36: 472-479, apr./dec 2004.

ABSTRACT - In the first part of this review, we discuss general strategies to treat exogenous intoxications (vomit induction, gastric lavage, administration of activated charcoal powder and of laxatives) and specific measures to eliminate toxic agents (forced diuresis and urine alkalinization, hemodialysis and hemoperfusion and utilization of antagonists). In the second part we discuss general principles of treatment of most prevalent intoxications in our area (agricultural pesticides, central nervous system depressants and rat poisons).

UNITERMS - Poisoning. Antidotes. Toxicology.

\section{BIBLIOGRAFIA CONSULTADA}

1 - GOLDFRANK LR, ed. Goldfrank's toxicologie emergencies. $7^{\text {th }}$.ed. McGraw-Hill Professional, New York, 2002.

2 - THOMSON MICROMEDEX. Poisindex - 1974-2003. Micromedex Health Series. www.periodicos.capes.gov.br

3 - MENEZES JB. Intoxicações agudas. Medicina, Ribeirão Preto, 25: 555-560, 1992.
4 - RANG HP \& DALE MM. Farmacologia. $4^{3}$ ed. Guanabara Koogan, Rio de Janeiro, 2001.

5 - KLAASSEN CD, ed. Casarett and Doull's toxicology: The basic science of poisons. $6^{\text {th }}$.ed. McGraw-Hill Medical Publication, New York, 2001.

6 - LING LJ \& CLARK RF. Toxicology secrets. Hanley-Belfus, Philadelphia, 2001. 